\title{
An In-Depth Study on Load Distribution Characteristics of the Planetary Threaded Roller Bearing
}

\author{
Shicheng Zheng $\mathbb{D}^{1}{ }^{1}$ Yongling Fu, ${ }^{1}$ Junlin Pan, ${ }^{1}$ Linjie Li, ${ }^{1}$ and Deyi Wang ${ }^{2}$ \\ ${ }^{1}$ School of Mechanical Engineering and Automation, Beihang University, Beijing 100083, China \\ ${ }^{2}$ Department National Key Laboratory of Science and Technology on Aerospace Intelligence Control, Beijing 100854, China
}

Correspondence should be addressed to Shicheng Zheng; shichengzhengs@163.com

Received 30 June 2020; Revised 21 September 2020; Accepted 28 October 2020; Published 10 November 2020

Academic Editor: Mitsuhiro Okayasu

Copyright (C) 2020 Shicheng Zheng et al. This is an open access article distributed under the Creative Commons Attribution License, which permits unrestricted use, distribution, and reproduction in any medium, provided the original work is properly cited.

\begin{abstract}
To lay a solid base for the property analysis and structural optimization on the high-strength planetary threaded roller bearing (PTRB) which is commonly used in mountain-walking robotic platforms, this research mainly focuses on characterizing the load distribution on the bearing under different loading conditions. An in-depth analysis was performed on the structural components and the contact characteristics of the PTRB. Based on the space meshing and Hertz contact theories, the elastic deformation of both inner and outer rings and the threaded roller was evaluated when the bearing was subjected to an axial or radial load. Meanwhile, a calculation method was proposed to obtain the load distribution coefficients of the PTRB according to the compatibility equations. In this way, an analysis on the load distribution of the PTRB was provided on the basis of the theoretical models. The results indicated that the effect of the applied load was very limited on the load distribution coefficients under an axial loading condition. The maximum value was found at the first thread of the inner ring-threaded roller contact pair, while no obvious effect was found when the bearing was carrying a radial load. It was indicated that the uniformity of the load distribution was effectively improved for axial and radial loading conditions through adjusting the distance between threads and increasing the threaded roller numbers, respectively. Therefore, both the rated load and the life cycle of the bearing can be further improved, while the friction torque can be minimized within a limited space. The research provides an important guidance for the property analysis, design, and optimization of the PTRB.
\end{abstract}

\section{Introduction}

Load distribution within the roller bearing is used to characterize the load differences between different contact points [1], which is one of the most important indexes for assessing the performance of the roller bearing since it has a direct effect on the rated load, life cycles, and friction torque of the bearing. And to improve the working performances of the mechanical systems, a study for the load distribution of the bearing is very helpful $[2,3]$. The contact characteristics of the PTRB can be obtained via both numerical calculation and experimental investigation based on Hertz contact theory and elastic mechanics $[4,5]$. Combined with the method of high calculation accuracy proposed by Jing Liu, load distribution and related contact characteristics can be analyzed $[2,3]$.
Guo and Li [6] and Cheng and Wang [7] investigated the load distribution within the tapered roller bearing and single-/double-row angular contact ball bearing, respectively. The relationship between the load distribution and the applied load was obtained, which provided a theoretical base for further investigations on the load distribution and structural optimization. However, the size deviation of the roller was neglected in these studies, which was different from the practical cases. Chen et al. [8] proposed a model to calculate the load distribution which took account of the effects of roller size deviation. Based on the size deviation induced by the manufacturing process, an in-depth research was carried out by Zhou and $\mathrm{Xu}$ [9] on the effects of size deviation magnitudes, the bearing clearance, the number of size-deviated rollers, and their locations on the load 
distribution within the bearing, which provide an essential support for the investigations on both static and fatigue behavior of the roller bearing.

Based on the structural features of the PTRB, the load distribution is not uniform between the thread teeth in the threaded connection [10]. Extensive studies, including both numerical and experimental ones, have been conducted in this field to discuss the effects of this ununiform load distribution $[11,12]$. Kenny and Patterson [13] studied the load distribution within the thread teeth via photoelastic tests and compared the experimental results with the theoretical model. Wang and Marshek [14] proposed a modified spring group model for the threaded connection and characterized the load distribution by using second-order difference equations. Sun and Liao [15] analyzed the axial load distribution within the low-sliding deformed thread connection via a symmetrical model and discussed the effects of the mesh density on the axial load distribution. In addition, Guo and Huang [16] investigated the load distribution on the thread teeth through a spring system with multi-degree-offreedom. A comparative study was performed on the load scenario on both nuts and screws under different loading conditions. A mathematical model was developed by Zhou and Sun $[17,18]$ by using a discretization method, and the axial load distribution on the thread teeth was numerically studied. Chen and Zeng [19] proposed a finite element model for characterizing the load distribution within the thread connection. The results on the load ratio between different thread teeth were validated by the experimental data. According to the structural characteristics of the casing thread joints, $\mathrm{Xi}$ and Nie [20] theoretically analyzed the effects of the pitch differences between inner and outer threads on the load transfer.

As a novel roller bearing, PTRB achieves a higher rated load and life cycle while lower friction torque in comparison to the traditional configuration. However, the ununiform load distribution during the loading process is still a limitation, which introduces difficulties in the property investigations. The research in this paper mainly analyzed the load distribution within the PTRB and put forward the analysis and calculation method of load distribution, which is commonly used in mountain-walking robotic platforms, under axial and radial loading conditions, which provide a theoretical support for the property studies on rated load, life cycles, and friction torque.

The outline of this paper is summarized as follows: Section 2 introduces the structural components and contact characteristics of the PTRB. The load distribution models for the axial and radial loading conditions are proposed in Sections 3 and 4, respectively. A detailed analysis on the load distribution of the PTRB is carried out in Section 5. Section 6 draws the main conclusions.

\section{Mechanical Structure and Contact Characteristics}

The mechanical structure of the PTRB is shown in Figure 1. The thread rollers are uniformly distributed outside of the inner ring throughout the entire process via matching of the holes designed on the planet carrier. The thread profile of the thread roller and of the inner (outer) ring are double circular and trapezoid, respectively. The thread roller meshes with the inner and outer ring. All these three parts have multiple annular threads, i.e., the thread stroke is equal to zero, with the same space.

PTRB can bear both axial and radial loads, as shown in Figure 2. When the PTRB bears an axial load, the side of the outer ring is fixed, and the inner ring bears the axial pressure transmitted by the actuator. Through the mutual contact of the threaded rollers with the outer ring and the inner ring, this axial load is transferred from the outer ring to the inner ring and eventually to the housing. The radial load is transmitted to the outer ring and the housing via the force threaded rollers. When the PTRB bears an axial load, each thread roller is stressed, and each thread tooth of each thread roller is only stressed on one side of the thread surface. When the PTRB bears a radial load, only part of the threaded roller is stressed, and each thread tooth of the stressed threaded roller is stressed on both sides of the thread surface.

The inner and outer ring-threaded roller contact pair were generated at the contact points. According to Hertz theory, two vertical planes can be obtained through the contact point of each contact pair, and both of them are perpendicular to the common tangent surface [21]. The radius of curvature of the inner ring-threaded roller contact pair in principle plane 1 is denoted as $R_{R 11}$ and $R_{I 21}$, while that in principle plane 2 is $R_{R 12}$ and $R_{I 22}$. Similarly, the radius of curvature of the outer ring-threaded roller contact pair in principle plane 1 is denoted as $R_{R 11}$ and $R_{O 21}$, while that in principle plane 2 is $R_{R 12}$ and $R_{\mathrm{O} 22}$. Figure 1 depicts the aforementioned parameters [22].

The contact characteristics of the bearing are directly linked to its structural parameters. The main structural parameters of the proposed PTRB are summarized in Table 1 and are marked in Figure 3.

\section{Load Distribution under the Axial Load}

The analysis on the local stress and deformation of each component in the PTRB is the premise of the investigation of the load distribution. This section mainly presents a study on the load distribution between thread teeth when the PTRB is subjected to an axial load according to the load-deformation relationship of the inner and outer rings and the threaded roller.

Despite of the effects of the applied load, the load distribution within the bearing is also influenced by the centrifugal force of the threaded roller and the gyroscopic moment during the running process. However, this influence can be neglected in this specific research owing to the low rotation velocity of the PTRB. That is to say, only the load distribution at static- or low-velocity running conditions is considered.

The following assumptions are made: (a) the load distribution analysis is performed considering only the axial loading condition. (b) Only elastic deformation takes place at the loading process, which satisfies Hooke's law. (c) The axial load is uniformly distributed at each threaded roller. (d) 


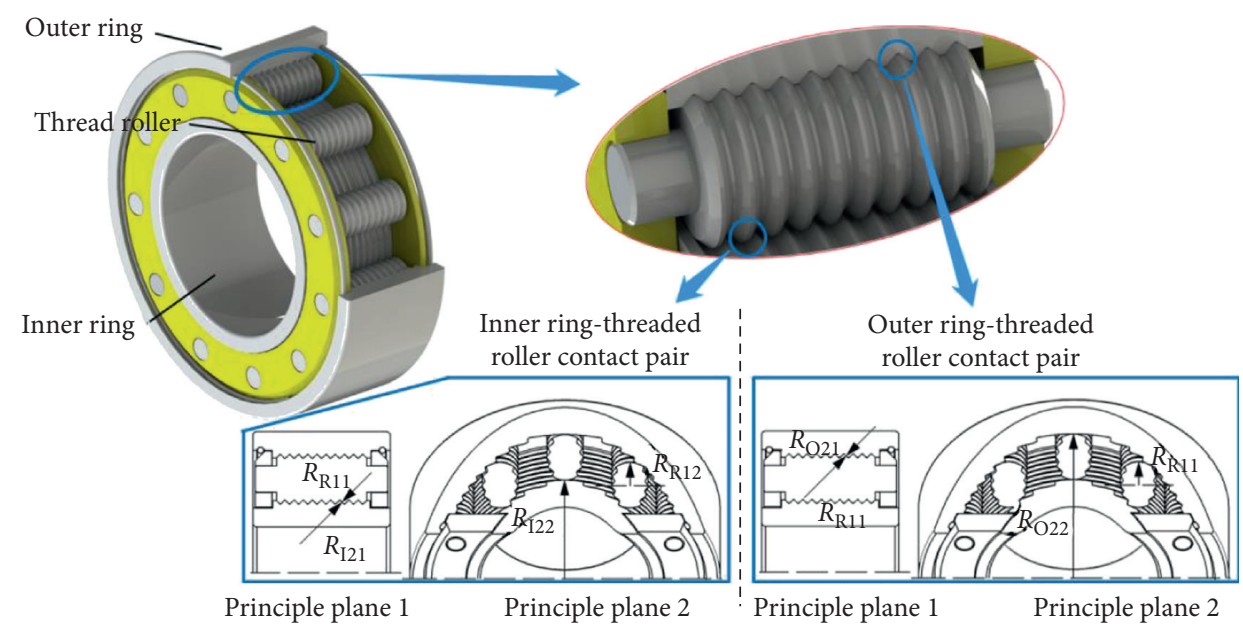

FIgURe 1: Structural diagram and contact characteristics of the PTRB.

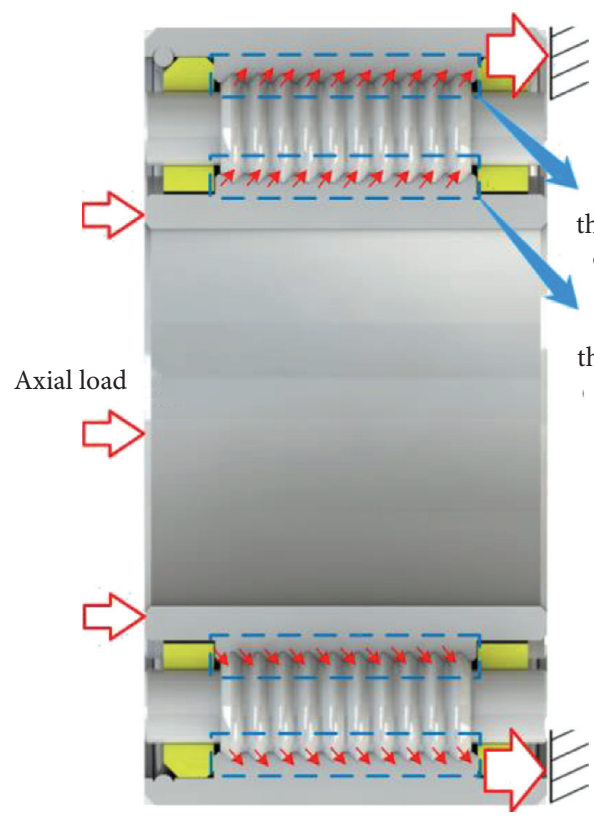

(a)

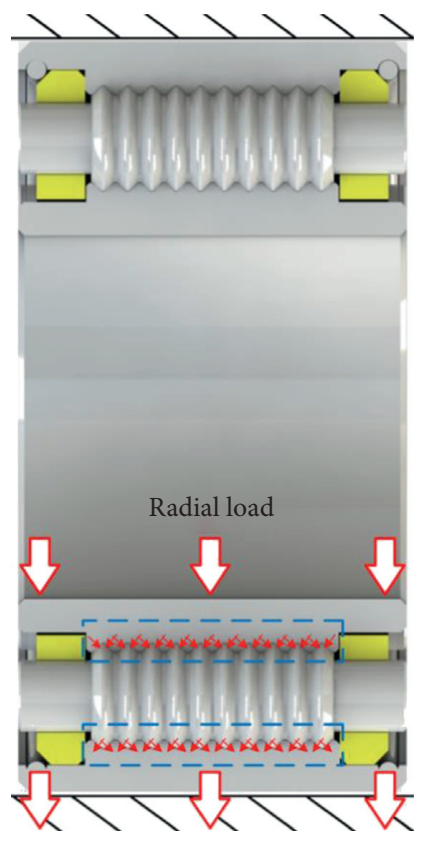

(b)

FIgURE 2: Schematic diagram of the force transfer path. (a) Transfer path of the axial force. (b) Transfer path of the radial force.

The contact surfaces are completely smooth, and the contact force is vertical to the surfaces.

3.1. Force Analysis and Elastic Deformation. A force decomposition was conducted at the contact points, as shown in Figure 4. It is obtained that the force components at the axial and vertical directions for the contact load $F_{\text {On }}$ and $F_{\text {In }}$, respectively, are expressed as

$$
\left\{\begin{array}{l}
F_{\text {Oai }}=F_{\text {Oni }} \sin \alpha, \\
F_{\text {Ori }}=F_{\text {Oni }} \cos \alpha, \\
F_{\text {Iai }}=F_{\text {Ini }} \sin \alpha, \\
F_{\text {Iri }}=F_{\text {Ini }} \cos \alpha .
\end{array}\right.
$$

The axial load applied at the PTRB is $F_{a}=Z \sum_{i=1}^{n} F_{\mathrm{Oai}}=$ $Z \sum_{i=1}^{n} F_{\text {Iai }}$ where $\alpha$ is the contact angle and $Z$ is the number of threaded rollers.

As indicated in Figure 4, the thread teeth of the roller are compressed and deformed by contacting those of both the inner and outer rings, which mainly present as the deformation of the thread teeth, the deformation of the shaft segment, and the contact deformation of the thread teeth at the contact points. These three typical deformations have a direct link to the ununiform load distribution at each contact point.

The first one represents the compressive or shear deformation of the thread teeth from the inner and outer rings and threaded roller caused by the applied load. The 
TABle 1: Main structural parameters of the PTRB.

\begin{tabular}{|c|c|c|}
\hline Parameter & Meaning of the parameters & Value \\
\hline$B_{I}$ & Thread bottom thickness of the inner ring & $1.08 \mathrm{~mm}$ \\
\hline$B_{O}$ & Thread bottom thickness of the outer ring & $1.08 \mathrm{~mm}$ \\
\hline$B_{R}$ & $\begin{array}{c}\text { Thread bottom thickness of the threaded } \\
\text { roller }\end{array}$ & $1.05 \mathrm{~mm}$ \\
\hline$s$ & Thread ridge thickness & $0.6 \mathrm{~mm}$ \\
\hline$h$ & Dedendum & $0.24 \mathrm{~mm}$ \\
\hline$d_{I}$ & Pitch diameter of $t$ & $36.5 \mathrm{~mm}$ \\
\hline$d_{O}$ & Pitch diameter of the bearing outer ring & $48.5 \mathrm{~mm}$ \\
\hline$d_{R}$ & Pitch diameter of the threaded roller & $6 \mathrm{~mm}$ \\
\hline$D_{0}$ & Outside diamet & $55 \mathrm{~mm}$ \\
\hline$d_{0}$ & of the PTRB & $30 \mathrm{~mm}$ \\
\hline$P$ & Distance between threads & $1.2 \mathrm{~mm}$ \\
\hline$\alpha$ & $\mathrm{Cc}$ & $45^{\circ}$ \\
\hline$\beta$ & Tooth & $45^{\circ}$ \\
\hline$E$ & Elasti & $212 \mathrm{GPa}$ \\
\hline$d_{\mathrm{rac}}$ & $\begin{array}{l}\text { Addendum circle diameter of the threaded } \\
\text { roller }\end{array}$ & $6.4 \mathrm{~mm}$ \\
\hline$\mu$ & Poissor & 0.29 \\
\hline$D_{\mathrm{pw}}$ & Pitch diameter of the thread pair & $42.5 \mathrm{~mm}$ \\
\hline$r_{\mathrm{I}}$ & Raceway radius of the bearing inner ring & $\infty$ \\
\hline$r_{\mathrm{O}}$ & Raceway radius of the bearing outer ring & $\infty$ \\
\hline$R_{r r}$ & Arc radius of the threaded roller & $5.1 \mathrm{~mm}$ \\
\hline$Z$ & Number of $\mathrm{t}$ & 11 \\
\hline$n$ & Number of thread teeth per threaded roller & 10 \\
\hline
\end{tabular}

deformation of the shaft segment is the compressive deformation of this structure under the compression load.
Note that the shaft segments are a ring cylinder for both inner and outer rings, while they are a solid cylinder for the threaded roller. The last one stands for the Hertz contact deformation caused by the contact force between the thread teeth. As a consequence, the total axial deformation $\left(\delta_{\text {Xai }}\right)$ at a specific point is the sum of the aforementioned three components:

$$
\delta_{\text {Xai }}=\delta_{\text {XTai }}+\delta_{\text {XAai }}+\delta_{\text {XCai }} \text {, }
$$

where $\delta_{\mathrm{XTai}}$ is the deformation of the thread teeth, $\delta_{\mathrm{XAai}}$ is the deformation of the shaft segment, and $\delta_{\mathrm{XCai}}$ is the contact deformation of the thread teeth.

3.2. Deformation of Thread Teeth. Both radial and axial deformation will take place once the thread teeth are subjected to a load normal to the contact surface. Only the axial deformation can be taken into consideration when analyzing the axial load distribution. The elastic deformation includes the bending deformation induced by the bending moment, the shear deformation induced by the shear force, the rotation deformation induced by the inclination of the tooth root, the sliding deformation induced by the shift of the tooth root, and the compressive deformation induced by the radial force components.

At a specific point, the axial deformation components of the thread teeth are denoted as $\delta_{\mathrm{XTani}}(n=1-5)$; thus, the total deformation is expressed as [23]

$$
\begin{aligned}
\delta_{\mathrm{XTai}}= & \delta_{\mathrm{XT} a 1 i}+\delta_{\mathrm{XT} a 2 i}+\delta_{\mathrm{XT} a 3 i}+\delta_{\mathrm{XT} a 4 i}+\delta_{\mathrm{XT} a 5 i} \\
= & \left(1-\mu^{2}\right) \frac{3 w_{X i} \cos \beta}{4 E}\left\{\left[1-\left(2-\frac{s}{B_{X}}\right)^{2}+2 \ln \left(\frac{B_{X}}{s}\right)\right] \cot ^{3} \beta-4\left(\frac{h}{B_{X}}\right)^{2} \tan \beta\right\} \\
& +(1+\mu) \frac{6 w_{\mathrm{X} i} \cos \beta}{5 E} \ln \left(\frac{B_{X}}{s}\right) \cot \beta+\left(1-\mu^{2}\right) \frac{12 h w_{X i} \cos \beta}{\pi E B_{X}^{2}}\left(h-\frac{s}{2} \tan \beta\right) \\
& +\left(1-\mu^{2}\right) \frac{2 w_{X i} \cos \beta}{\pi E}\left[\frac{P}{B_{X}} \cdot \ln \left(\frac{P+\left(B_{X} / 2\right)}{P-\left(B_{X} / 2\right)}\right)+\frac{1}{2} \ln \left(\frac{4 P^{2}}{B_{X}^{2}}-1\right)\right]+\delta_{\mathrm{XT} a 5 i},
\end{aligned}
$$

where $w_{X i}$ and $\beta$ are the unit force of the normal load at the contact point and the half-angle of the thread teeth, $\mu$ is Poisson's ratio, $P$ is the distance between threads, $E$ is the elastic modulus, $h$ is the dedendum, and $s$ is the thread ridge thickness.

For different thread configurations, the deformation of the thread teeth induced by the radial force components is different. The deformation for the inner ring with outer threads is [23]

$$
\delta_{\mathrm{IT} a 5 i}=\left(\frac{d_{I}^{2}+d_{0}^{2}}{d_{I}^{2}-d_{0}^{2}}-\mu\right) \frac{d_{I} w_{X i}^{\prime} \sin \beta}{2 P E} \tan \beta .
$$

The deformation for the outer ring with inner threads is [23]

$$
\delta_{\mathrm{RT} a 5 i}=(1-\mu) \frac{d_{R} w_{X i}^{\prime} \sin \beta}{2 P E} \tan \beta .
$$

The deformation for the threaded roller with outer threads is [23]

$$
\delta_{\text {OTa5i }}=\left(\frac{D_{0}^{2}+d_{O}^{2}}{D_{0}^{2}-d_{O}^{2}}+\mu\right) \frac{d_{\mathrm{O}} w_{X i}^{\prime} \sin \beta}{2 P E} \tan \beta,
$$

where $w_{X i}^{\prime}$ is the unit force of the normal load at the circumference of the thread, $d_{I}$ is the pitch diameter of the bearing inner ring, $d_{O}$ is the pitch diameter of the bearing outer ring, $D_{0}$ is the outside diameter of the PTRB, and $d_{0}$ is the inner diameter of the PTRB. 


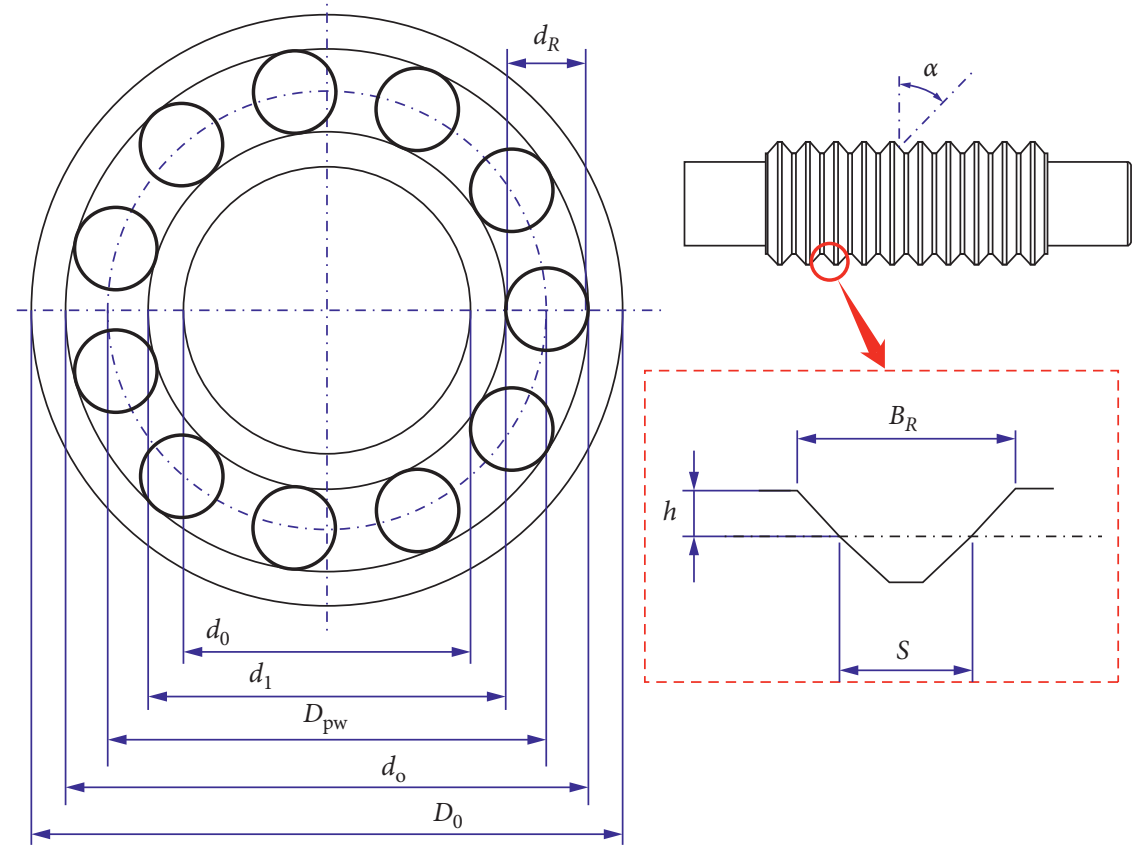

Figure 3: Main structural parameters of the PTRB.

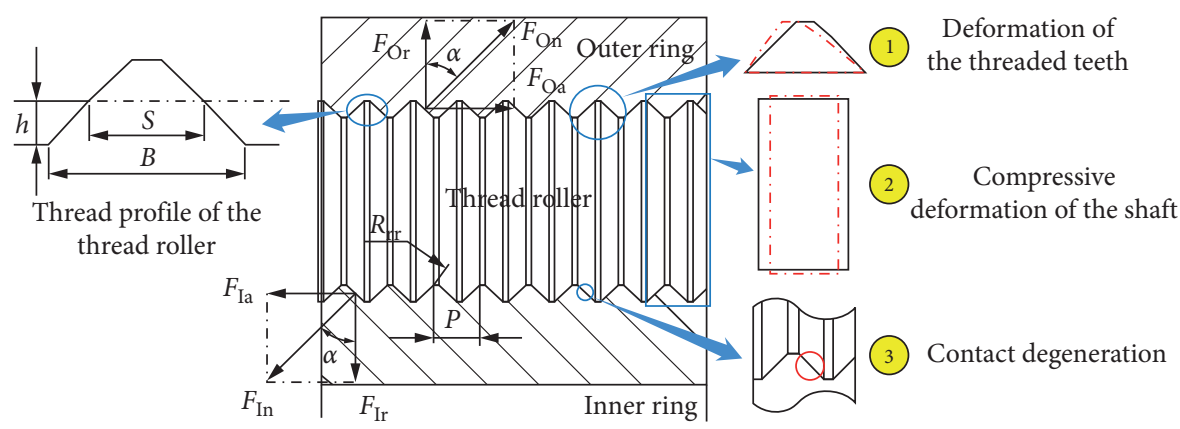

Figure 4: Schematic diagram of the force decomposition and elastic deformation of the PTRB.

3.3. Deformation of the Shaft Segment. Owing to the assumptions on continuity, uniformity, isotropy, and small deformation, the deformation of the shaft segment can be obtained by using the tension-compression stiffness formula in material mechanics [24]:

$$
\delta_{\text {XAai }}=\frac{F_{\mathrm{Xai}} P}{E A_{X}} .
$$

Note that the number for the contact points in the inner and outer ring and the threaded roller is increasing from left to right. Consequently, the numbers for the contact points in the inner and outer rings are from 1 to $n$, while for the threaded roller, they are from 1 to $2 n$. The components are divided into several segments by the contact points. The corresponding numbers for the segments of the inner and outer ring are from 0 to $n$, while for those of the threaded roller are from 0 to $2 n$. The axial load and cross-sectional area for each segment of the inner ring can be expressed as

$$
\begin{aligned}
& F_{\text {Iai }}= \begin{cases}-\frac{F_{a}}{Z}, & i=0, \\
-\frac{F_{a}}{Z}+\sum_{j=1}^{i} F_{\text {Iaj }}, & i>0,\end{cases} \\
& A_{I}=\frac{\pi\left[\left(d_{I}-2 h\right)^{2}-d_{0}^{2}\right]}{4 Z} .
\end{aligned}
$$

These values for the segments of the outer ring are

$$
\begin{gathered}
F_{\text {Oai }}= \begin{cases}0, & i=0, \\
-\sum_{j=1}^{i} F_{\text {Oaj }}, & i>0,\end{cases} \\
A_{O}=\frac{\pi\left[D_{0}^{2}-\left(d_{O}+2 h\right)^{2}\right]}{4 Z} .
\end{gathered}
$$


These values for the segments of the threaded roller are

$$
\begin{aligned}
& F_{\text {Rai }}= \begin{cases}0, & i=0, \\
-F_{\text {Ial }}, & i=1, \\
\sum_{j=1}^{k}\left(F_{\text {Oaj }}-F_{\text {Iaj }}\right), & i=2 k(k>0), \\
\sum_{j=1}^{k}\left(F_{\text {Oaj }}-F_{\text {Iaj }}\right)-F_{I a(k+1)}, & i=2 k+1(k>0),\end{cases} \\
& A_{R}=\frac{\pi\left(d_{R}-2 h\right)^{2}}{4}
\end{aligned}
$$

where $d_{R}$ is the pitch diameter of the threaded roller and $Z$ is the number of threaded rollers.

3.4. Contact Deformation of Thread Teeth. Owing to the mismatch of the thread teeth of the threaded roller (doublecircular shape) and of the inner and outer rings (trapezoidal shape), it is point contact for the surfaces of both inner and outer ring-threaded roller pairs. The contact deformation is lying at the normal line direction of their common tangent surface.

The curvature is the reciprocal of the curvature radius. Therefore, according to Figure 1, the curvature at the contact point of the inner ring-threaded roller pair is obtained as [1]

$$
\left\{\begin{array}{l}
\rho_{I 11}=\frac{1}{R_{R 11}}=\frac{1}{R_{\mathrm{rr}}}, \\
\rho_{I 12}=\frac{1}{R_{R 12}}=\frac{2 \cos \alpha}{d_{R}}, \\
\rho_{I 21}=\frac{1}{R_{I 21}}=-\frac{1}{r_{I}}, \\
\rho_{I 22}=\frac{1}{R_{I 22}}=\frac{2 \cos \alpha}{d_{I}} .
\end{array}\right.
$$

Similarly, the curvature value of the outer ring-threaded roller pair is

$$
\left\{\begin{array}{l}
\rho_{\mathrm{O} 11}=\frac{1}{R_{R 11}}=\frac{1}{R_{\mathrm{rr}}}, \\
\rho_{\mathrm{O} 12}=\frac{1}{R_{R 12}}=\frac{2 \cos \alpha}{d_{R}}, \\
\rho_{\mathrm{O} 21}=\frac{1}{R_{\mathrm{O} 21}}=-\frac{1}{r_{O}}, \\
\rho_{\mathrm{O} 22}=\frac{1}{R_{\mathrm{O} 22}}=-\frac{2 \cos \alpha}{d_{O}},
\end{array}\right.
$$

where $R_{\mathrm{rr}}$ is the arc radius of the threaded roller, $r_{I}$ is the raceway radius of the bearing inner ring, and $r_{O}$ is the raceway radius of the bearing outer ring.

The sum of the main curvature values at the contact point is an intrinsic characteristic of a contact pair. The sum of the main curvature for both inner $\left(\left(\sum \rho\right)_{\mathrm{IR}}\right)$ and outer $\left(\left(\sum \rho\right)_{\mathrm{OR}}\right)$ ring-threaded roller pairs can be calculated by using equations (10) and (11), respectively. The main curvature function for two types of contact pairs is [1]

$$
\left\{\begin{array}{l}
F_{\mathrm{IR}}(\rho)=\frac{\left|\left(\rho_{\mathrm{I} 11}-\rho_{\mathrm{I} 12}\right)+\left(\rho_{I 21}-\rho_{I 22}\right)\right|}{\left(\sum \rho\right)_{\mathrm{IR}}}, \\
F_{\mathrm{OR}}(\rho)=\frac{\left|\left(\rho_{\mathrm{O} 11}-\rho_{\mathrm{O} 12}\right)+\left(\rho_{\mathrm{O} 21}-\rho_{\mathrm{O} 22}\right)\right|}{\left(\sum \rho\right)_{\mathrm{OR}}} .
\end{array}\right.
$$

Based on the Hertz contact formula, the contact deformation of thread teeth can be obtained by projecting the deformation at the contact point of thread teeth pairs under the normal contact load to the axial direction [1]:

$$
\delta_{\mathrm{XCai}}=\frac{2 K(e)}{\pi m_{a X}} \sqrt[3]{\frac{9}{8}\left(\frac{1-\mu^{2}}{E}\right)^{2} F_{X \mathrm{ni}}^{2}\left(\sum \rho\right)_{X}} \sin \alpha,
$$

where the value of the contact elliptic coefficient $\left(2 K(\mathrm{e}) / \pi m_{\mathrm{aX}}\right)$ can be obtained by using the lookup method based on the main curvature function.

3.5. Compatibility Equations. According to the equilibrium condition of forces, the equilibrium equations of the PTRB at the axial direction are denoted as

$$
\left\{\begin{array}{l}
Z \sum F_{\text {Iai }}=F_{a} \\
Z \sum F_{\text {Oai }}=F_{a} .
\end{array}\right.
$$

Assuming that each contact point keeps contacting throughout the loading process, the deformation of the thread teeth for the inner ring-threaded roller pair is

$$
\begin{gathered}
\left(\delta_{\mathrm{IA} a}\right)_{i}+\left(\delta_{\mathrm{IT} a}\right)_{i}-\left(\delta_{\mathrm{IT} a}\right)_{i+1}+\left(\delta_{\mathrm{IRC} a}\right)_{i}-\left(\delta_{\mathrm{IRC} a}\right)_{i+1} \\
=\left(\delta_{\mathrm{RA} a}\right)_{2 i-1}+\left(\delta_{\mathrm{RA} a}\right)_{2 i}-\left(\delta_{\mathrm{RT} a}\right)_{2 i-1}+\left(\delta_{\mathrm{RT} a}\right)_{2 i+1} .
\end{gathered}
$$

Similarly, the deformation of the thread teeth for the inner ring-threaded roller pair is

$$
\begin{gathered}
\left(\delta_{\mathrm{OA} a}\right)_{i}-\left(\delta_{\mathrm{OT} a}\right)_{i}+\left(\delta_{\mathrm{OT} a}\right)_{i+1}-\left(\delta_{\mathrm{ORC} a}\right)_{i}+\left(\delta_{\mathrm{ORC} a}\right)_{i+1} \\
=\left(\delta_{\mathrm{RA} a}\right)_{2 i}+\left(\delta_{\mathrm{RA} a}\right)_{2 i+1}+\left(\delta_{\mathrm{RT} a}\right)_{2 i}-\left(\delta_{\mathrm{RT} a}\right)_{2 i+2} .
\end{gathered}
$$

There are $2 n$ unknown contact forces at the inner and outer rings contacted by a threaded roller. Owing to the two equilibrium equations in equation (15), there are $2 n-2$ compatibility equations in total in equations (16) and (17). Combining the equations from (1) to (17), we can use MATLAB to solve the equations to solve the load distribution of the PTRB along the axial direction.

For the sake of the modelling and analysis on other indexes, such as rated load, friction, and life cycle, the axial 
load distribution coefficient $f_{\text {ai }}$ is defined as the ratio between the axial load of each thread and the averaged axial load:

$$
f_{\mathrm{ai}}=\frac{n Z F_{\mathrm{Xai}}}{F_{a}}
$$

where $n$ is the number of thread teeth per threaded roller.

\section{Load Distribution under the Radial Load}

Once the PTRB is only subjected to a radial load, it is necessary to conduct an analysis on the load distribution due to different deformations caused by different contact forces at different positions of the threaded roller. This section mainly presents a study on the load distribution between thread teeth when the PTRB is subjected to a radial load according to the load-deformation relationship of the inner and outer rings and the threaded roller, which provides a support for the structural design and property investigations. Note that the assumptions made for analysis under the axial load are also provided in this section for the specific loading condition, i.e., radial load.

4.1. Force Analysis and Elastic Deformation. As depicted in Figures 2 and 5, only half of the threaded roller are subjected to the contact force under the radial load at the vertical direction. The part that bears contact force is defined as an effective threaded roller. Therefore, the number of the effective threaded rollers $\left(Z_{e}\right)$ during the rotation process is

$$
\begin{aligned}
Z_{e} & =\left[\frac{Z-1}{2}\right], \\
\text { or } Z_{e} & =\left[\frac{Z+1}{2}\right] .
\end{aligned}
$$

The symbol [] in equation (19) is a Gauss mark, that is, $[x]$ can be used to represent the largest integer not exceeding $x$, for example, $[1.2]=1$.

Defining the angle between the first effective threaded roller from the left side and the horizontal line is $\theta_{0}$, the argument $\left(\varphi_{i}\right)$ of each effective threaded roller is denoted as

$$
\varphi_{i}=\theta_{0}+(i-1) \frac{2 \pi}{Z}, \quad \theta_{0} \in\left(0, \frac{2 \pi}{Z}\right] \text {. }
$$

The force equilibrium equation can be obtained via Newton's third law:

$$
2 n \sum_{i=1}^{Z_{e}} F_{r i} \sin \left(\varphi_{i}\right)=F_{r}
$$

Similar to the axial loading condition, the bearing deformation consists of the deformation of the contact points and the thread teeth under the radial load. The deformation of the shaft segment can be neglected owing to its significant high stiffness.

4.2. Elastic Deformation of Thread Teeth. According to the analysis on Figure 2, owing to the geometric symmetry, the

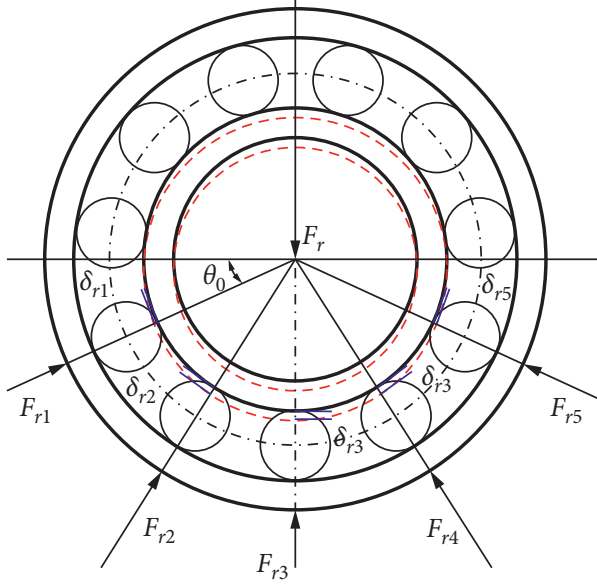

FIGURE 5: Schematic diagram of the load distribution at the radial direction.

deformation induced by bending, shear, rotation, and sliding are all offset when the PTRB is subjected to a radial load. Therefore, only the compressive deformation $\delta_{\mathrm{XTri}}$ caused by the radial force component is considered.

For the inner threads at the inner ring, the deformation is expressed as [23]

$$
\delta_{\mathrm{ITri}}=\left(\frac{d_{I}^{2}+d_{0}^{2}}{d_{I}^{2}-d_{0}^{2}}-\mu\right) \frac{d_{I} w_{X i}^{\prime} \sin \beta}{2 \mathrm{PE}} .
$$

For the outer threads at the outer ring [23],

$$
\delta_{\mathrm{OTri}}=\left(\frac{D_{0}^{2}+d_{O}^{2}}{D_{0}^{2}-d_{O}^{2}}+\mu\right) \frac{d_{O} w_{X i}^{\prime} \sin \beta}{2 \mathrm{PE}} .
$$

For the outer threads at the threaded roller [23],

$$
\delta_{\mathrm{RTri}}=(1-\mu) \frac{d_{R} w_{X i}^{\prime} \sin \beta}{2 \mathrm{PE}} .
$$

Thus, the total deformation is denoted as

$$
\delta_{\mathrm{Tri}}=\delta_{\mathrm{ITri}}+\delta_{\mathrm{OTri}}+2 \delta_{\mathrm{RTri}} \text {. }
$$

4.3. Contact Deformation of Thread Teeth. Similar to the deformation occurring in the thread teeth under the axial load, the contact deformation can be obtained by projecting the deformation at the contact point of thread teeth pairs under the normal contact load to the radial direction [1]:

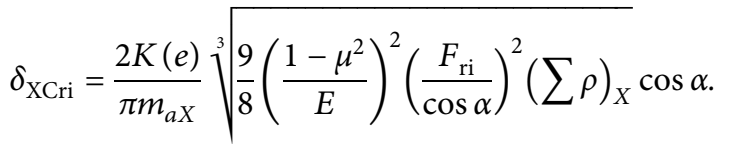

Then, the total contact deformation of the thread teeth is

$$
\delta_{\text {Cri }}=\delta_{\text {IRCri }}+\delta_{\text {ORCri }} .
$$

4.4. Compatibility Equations. As indicated in Figure 5, the vertical deformations of two adjacent effective threaded rollers are equal, and we can get 


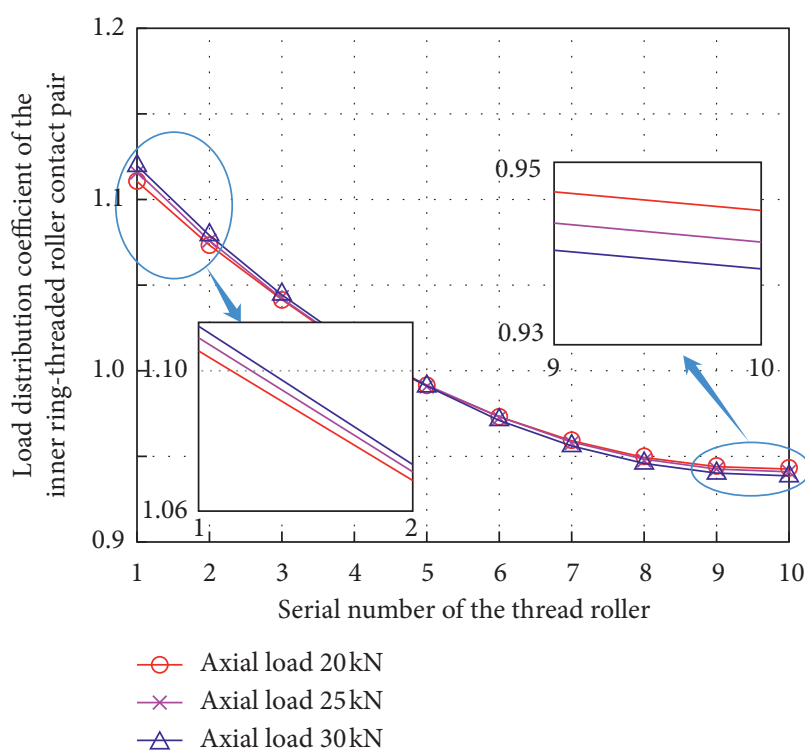

(a)

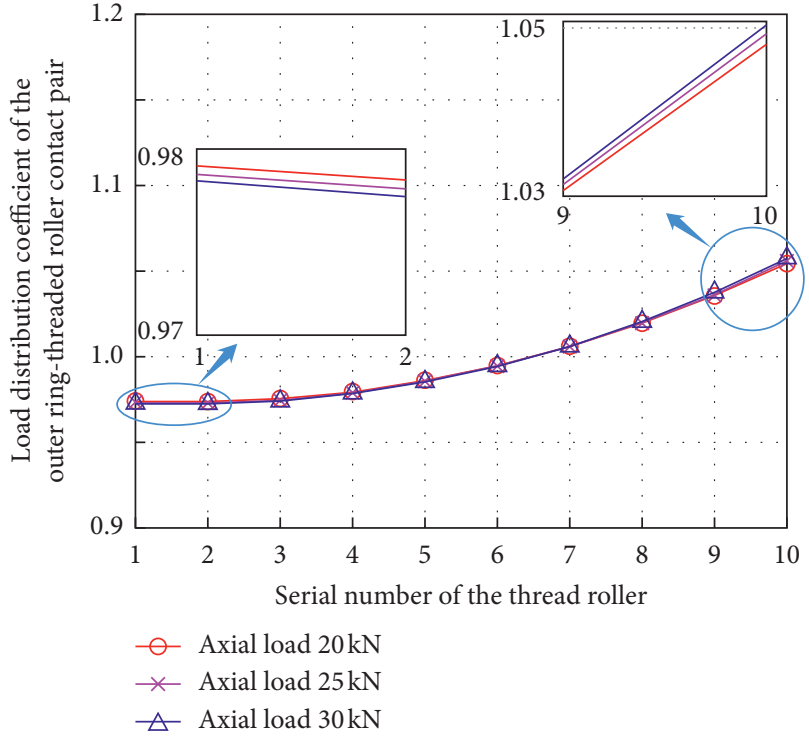

(b)

Figure 6: Evolution of the axial load distribution coefficient with the axial load.

$$
\frac{\delta_{r 1}}{\sin \varphi_{1}}=\frac{\delta_{r 2}}{\sin \varphi_{2}}=\frac{\delta_{r 3}}{\sin \varphi_{3}}=\frac{\delta_{r 4}}{\sin \varphi_{4}}=\frac{\delta_{r 5}}{\sin \varphi_{5}} .
$$

So, according to the equal displacement of two adjacent effective thread rollers in the vertical direction, as a deformation compatibility equation,

$$
\frac{\left(\delta_{C r i}+\delta_{T r i}\right)}{\sin \varphi_{i}}=\frac{\left(\delta_{C r(i+1)}+\delta_{T r(i+1)}\right)}{\sin \varphi_{(i+1)}} .
$$

There are $Z_{e}$ unknown contact forces by a threaded roller. Owing to the equilibrium equation in equation (21), there are $Z_{e}-1$ compatibility equations in total in equation (29). Combining the equations from (19) to (29), the load distribution in the PTRB along the axial direction can be solved by using MATLAB.

For the sake of the modelling and analysis on other indexes, such as rated load, friction, and life cycle, the radial load distribution coefficient $f_{r \varphi i}$ is defined as the ratio between the radial load of each thread and the axial load carried by the entire bearing:

$$
f_{r \varphi i}=\frac{2 n F\left(\varphi_{i}\right)}{F_{r}}, \quad \varphi_{i} \in[0,2 \pi),
$$

where $F\left(\varphi_{i}\right)$ is the radial force at a random contact point on the threaded roller at the location of $\varphi_{i}$.

\section{Analysis on the Load Distribution}

5.1. The Axial Load Case. Based on the theoretical analysis, the evolution of the load distribution as a function of the axial load was obtained by using MATLAB. As indicated in Figure 6, the load distribution did not show a significant change when increasing the applied load, which implied that the axial load distribution coefficient can be regarded as an inherent characteristic of the PTRB with a certain structural configuration. However, the load distribution coefficients of inner and outer ring-threaded roller pairs showed different magnitudes and contradictory evolution trends, ranging $0.94 \sim 1.12$ for the former and $0.97 \sim 1.05$ for the latter. It can be seen that the inner ring-threaded roller pair showed less uniformity in the load distribution.

As a result of the further analysis, when given an axial load of $30 \mathrm{kN}$, the spacing between every two adjacent contact points was leveling at around $0.1 \mu \mathrm{m}$ as comparing to the difference in the tooth spacing $(1.2 \mathrm{~mm})$, which indicated that the ununiform load distribution was caused by this tiny difference in the tooth spacing. Therefore, one can adjust the spacing between two contact points according to the deformation coordination, thus minimizing the ununiformity of the load distributed on the thread teeth. This will be done in the future work.

5.2. The Radial Load Case. Similarly, the evolution of the load distribution as a function of the radial load was obtained by using MATLAB.

As illustrated in Figure 7, the maximum load distribution coefficient on the radial direction of the PTRB was 0.373. The applied load had little effect on the load distribution, which indicated that the radial load distribution coefficient can also be regarded as an inherent characteristic of the PTRB with a certain structural configuration.

Figure 8 illustrates the evolution of the radial load distribution as a function of the number of the threaded rollers on the basis of a fixed structural configuration of the PTRB. When the number of the threaded rollers increased from 9 to 11, the maximum load distribution coefficient decreased from 0.460 to 0.373 , which revealed that a higher radial load uniformity can be achieved by increasing the number of thread rollers. From a viewpoint of structural 




Figure 7: Evolution of the radial load distribution coefficient with the radial load.



_ Number of thread rollers: 9

- Number of thread rollers: 10

_ Number of thread rollers: 11

FIGURE 8: Evolution of the radial load distribution with the number of threaded rollers.

design, given an increased threaded roller number, the load at each contact point is reduced, which tends to improve the rated load and life cycles for the bearing. In addition, an empirical study indicates that the friction torque can be lower combined with a neglected influence on the original bearing structure when properly increasing the number of threaded rollers, which therefore should be considered in priority [25].

However, it should be noted that the number of threaded rollers is limited by the installation space and the installation methods. Figure 9 demonstrates the PTRB with the most

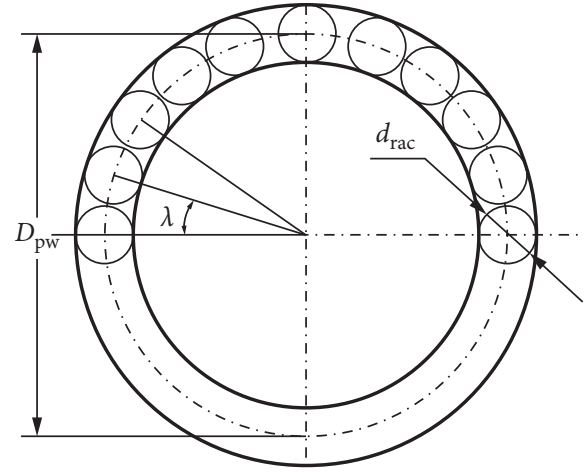

FIGURE 9: Installation and distribution of the threaded rollers.

threaded rollers inserted. There are 11 threaded rollers in total which are uniformly distributed at the circumference of the inner ring, which results in an angle $\lambda$ in between each threaded roller pair.

The relationship between the maximum number of the threaded rollers and the PTRB structural parameters is expressed as

$$
Z \leq\left\lfloor\frac{\pi / 2}{\left.\arcsin \left(d_{\mathrm{rac}} / D_{\mathrm{pw}}\right)\right\rfloor+1,}\right.
$$

where $d_{\mathrm{rac}}$ is the diameter of the addendum circle in the threaded roller.

\section{Conclusions}

Load distribution within the PTRB has vital importance on its property analysis and structural optimization. The research in this paper mainly focuses on the load distribution of the PTRB used in the mountain-walking robotic platform.

(1) An introduction was provided for the mechanical structures and the load transfer within the PTRB. A detailed analysis was performed regarding the contact patterns of both inner and outer ring-threaded roller pairs. A mathematical model was proposed on the basis of the elastic deformation and the force nonlinearity of each major load-carrying part, providing a support for the load distribution analysis.

(2) The load distribution coefficients of the PTRB were calculated by using the compatibility equations and MATLAB. The load distribution analysis results indicated that the applied load had neglected effect on the load distribution coefficients, which indicated that a set of approximation values can be regarded as the coefficients for a PTRB with a fixed structural configuration. The axial load distribution coefficients showed different magnitudes and contradictory trends for the inner and outer ring-threaded roller pairs. The values on the radial direction were different but radially symmetrical at different locations of the threaded roller.

(3) The uniformity of the load distribution on the axial and radial directions can be improved by adjusting 
the distance between threads and increasing the number of threaded rollers, respectively. When the roller number was increased to 11 , the maximum load coefficient decreased to 0.373 .

\section{Data Availability}

The data used to support the findings of this study are available from the corresponding author upon request.

\section{Conflicts of Interest}

The authors declare that they have no conflicts of interest.

\section{References}

[1] C. Wan, Analysis Method of Rolling Bearing, Machinery Industry Press, Beijing, China, 1987.

[2] J. Liu, "A dynamic modelling method of a rotor-roller bearing-housing system with a localized fault including the additional excitation zone," Journal of Sound and Vibration, vol. 469, Article ID 115144, 2019.

[3] J. Liu, C. Tang, H. Wu et al., "An analytical calculation method of the load distribution and stiffness of an angular contact ball bearing," Mechanism and Machine Theory, vol. 142, Article ID 103597, 2019.

[4] Z. Niu, Performance Analysis of Thin-Wall Crossed Cylindrical Roller Bearings, Henan University of Science and Technology, Luoyang, China, 2018.

[5] W. Zhang, Model and Method for Calculating the Load Distribution of the Thread Teeth of Planetary Roller Screw Pairs, Northwestern Polytechnical University, Xi'an, China, 2017.

[6] H. Guo and Y. Li, "Load distribution analysis of angular contact ball bearings and tapered roller bearings," Mechanical Design, vol. 35, no. S2, pp. 194-196, 2018.

[7] C. Cheng and J. Wang, "Load analysis of double-row angular contact ball bearings," Mechanical Science and Technology, vol. 33, no. 6, pp. 829-833, 2014.

[8] G. Chen, F. Mao, and B. Wang, "Effects of off-sized cylindrical rollers on the static load distribution in a cylinder roller bearing," Proceedings of the Institution of Mechanical Engineers, Part J: Journal of Engineering Tribology, vol. 226, no. 8, pp. 687-696, 2012.

[9] X. Zhou and H. Xu, "Research on load distribution of angular contact ball bearings under dimensional deviation," $\mathrm{Me}$ chanical Transmission, vol. 38, no. 3, pp. 35-40, 2014.

[10] L. Pu and M. Ji, Mechanical Design, Higher Education Press, Beijing, China, 2006.

[11] X. Hu and H. Lu, "Research of loading distribution of nut made by phenolic resin in spiral driver," Chinese Journal of Mechanical Engineering, vol. 40, no. 04, pp. 185-189, 2004.

[12] Z. Huang and J. Ren, "Research on the stress and working pressure of screw teeth in sliding screw transmission," Forging Technology, vol. 29, no. 02, pp. 56-59, 2004.

[13] B. Kenny and E. A. Patterson, "Load and stress distribution in screw threads," Experimental Mechanics, vol. 25, no. 3, pp. 1-6, 1985.

[14] W. Wang and K. M. Marshek, "Determination of load distribution in a threaded connector with yielding threads," Mechanism and Machine Theory, vol. 31, no. 2, pp. 229-244, 1996.
[15] Y. Sun and R. Liao, "Research on axial load and stress distribution of threaded couplings," National Annual Conference of High Power Diesel Engines, vol. 29, 2007.

[16] W. Guo and W. Huang, "Thread force distribution analysis method and its application examples," Science and Technology Information, vol. 17, pp. 70-71, 2010.

[17] X. Zhou and Y. Sun, "Spring group model of axial force distribution of transmission screw thread," Mechanical Design, vol. 24, no. 6, pp. 30-33, 2007.

[18] X. Zhou and Y. Sun, "Analysis of the axial load distribution law of transmission screw thread based on finite element method," Mechanical Design and Manufacturing, vol. 1, pp. 16-18, 2008.

[19] H. Chen and P. Zeng, "Load distribution of bolted joint," Journal of Mechanical Engineering, vol. 46, no. 09, pp. 171178,2010

[20] J. Xi and G. Nie, "The effect of pitch error on the load transfer characteristics of casing thread," Journal of Xi'an Jiaotong University, vol. 1, pp. 46-49, 2000.

[21] D. Olaru and G. C. Puiu, A New Model to Estimate Friction Torque in a Ball Screw System. Product Engineering, Springer, Dordrecht, Netherlands, 2004.

[22] F. Lisowski, "Nmnerical computation of stresses and deformations in the planetary roller screw components," Technical Transactions, vol. 7, pp. 141-148, 2015.

[23] H. Yamamoto, Theory and Calculation of Threaded Connection, Shanghai Science and Technology Literature Press, Shanghai, China, 1984.

[24] H. Liu, Material Mechanics, Higher Education Press, Beijing, China, 2004.

[25] S. Deng and Y. Jia, "Research on friction torque characteristics of thrust ball bearings," Journal of Ordnance Engineering, vol. 9, pp. 17-25, 2015. 